\title{
Importancia de los microcréditos como instrumento de financiación en el desarrollo social*
}

\author{
María Elena Inglada Galiana,' José Manuel Sastre Centeno² \\ y María Cristina de Miguel Bilbao ${ }^{3}$ \\ Universidad de Valladolid (España)
}

Recibido: junio 15 de 2015 - Revisado: agosto 6 de 2015 - Aceptado: octubre 20 de 2015

Referencia formato APA: Inglada-Galiana, M. E., Sastre-Centeno, J. M., \& De Miguel-Bilbao, M. C. (2015). Importancia de los microcréditos como instrumento de financiación en el desarrollo social. Revista Cientifica Guillermo de Ockham, 13(2), 89-100.

\section{Resumen}

El éxito del desarrollo de las microfinanzas en general y los microcréditos en particular entre las clases más pobres de los países menos desarrollados, ha cambiado los parámetros sobre los tipos de ayudas a dichos colectivos. Por otra parte, la evolución de las microfinanzas durante las últimas tres décadas ha supuesto un cambio en aquellas estructuras que se han profesionalizado, alejándose así de los subsidios estatales y acercándose a la autofinanciación y, consecuentemente, al mercado financiero tradicional, aunque sin abandonar las características básica del sector. En este trabajo pretendemos explicar el porqué de las microfinanzas y los microcréditos, sus objetivos fundamentales, la estructura operativa y el mercado, humano y social en el que se desarrollan sus actividades. Pretendemos asimismo, establecer una pauta de comportamiento y acción de la coyuntura actual del sector y vislumbrar una proyección futura, que permita conocer si las microfinanzas tienden a una mayor expansión o, por el contrario, los problemas con que se enfrenta pueden ralentizar su desarrollo actual. El hecho de que los microcréditos hayan traspasado las fronteras de los países pobres y se estén implantando también en los más desarrollados, solo enfatiza el éxito de los mismos y que las bolsas de pobreza están presentes en cualquier lugar del mundo. El futuro de los microcréditos está tanto en los países en vías de desarrollo como en los del primer mundo.

Palabras clave: Pobreza, necesidad, empoderamiento, acción social, subdesarrollo, micronegocios

\section{Importance of microcredit as a tool for social development financing}

\section{Abstract}

The successful development of microfinance in general and microcredits in particular among the poorest classes of the least developed countries, has changed the parameters on the types of support to these groups. In this paper, we explain why microfinance and microcredits, its fundamental objectives, operational and market structure, human and social, in which its activities are carried out. We intend to establish a pattern of behaviour and action of the current

* Este trabajo es financiado por el Departamento de contabilidad de la Universidad de Valladolid (España) en la linea de investigación de economía social.

1. Doctora por la Universidad de Valladolid dentro del Programa de Doctorado en Gestión y Administración de Empresas. En la actualidad es Profesora y Coordinadora de Sección en el Departamento de Economía Financiera y Contabilidad de la Universidad de Valladolid. Dirección postal: Facultad de CC.EE. Y Empresariales. Avda. del valle Esgueva 6 47011. E-mail: elenaig@eco.uva.es

2. Doctor por la Universidad de Valladolid dentro del Programa de Doctorado en Gestión y Administración de Empresas. En la actualidad es Profesor Ayudante Doctor en el Departamento de Economía Financiera y Contabilidad de la Universidad de Valladolid. E-mail: manolo@eco.uva.es

3. Doctora por la Universidad de Valladolid dentro del Programa de Doctorado en Gestión y Administración de Empresas. En la actualidad es Profesora Contratada Doctora en el Departamento de Economía Financiera y Contabilidad de la Universidad de Valladolid. E-mail: cdmiguel@efc.uva.es 
situation of the sector and envision a future projection, designed to show whether microfinance tend to further expansion or, on the contrary, the problems faced by the current development may slow. The analysis follows that the system needs to be funded to be effective, covering the high costs thereof. Therefore, it has been necessary to resort to external financing, mainly through large capitalist conglomerates, which can ensure the necessary funding for further expansion to decrease the high fixed costs of microloans. In achieving this objective, which allows to produce benefits and capitalized largely depend on the survival of this financing system.

Keywords: Poverty, need, empowerment, social action, underdevelopment, microbusinesses

\section{Importância do microcréditos com uma ferramenta para o financiamento do desenvolvimento social}

\section{Resumo}

O êxito do desenvolvimento de microfinanças e microcrédito, em -em geral especialmente entre as classes mais pobres dos países menos desenvolvidos, mudou os parâmetros dos tipos de ajuda a estes grupos. Este artigo explica por que as microfinanças eo microcrédito, os seus principais objectivos, estrutura operacional e de mercado, humano e social, em que as suas actividades são realizadas. Temos a intenção de estabelecer um padrão de comportamento e ação da situação actual do sector e prever uma projeção futura, que permite saber se as microfinanças tendem a uma maior expansáo ou, pelo contrário, os problemas enfrentados seu desenvolvimento atual pode atrasar. A análise conclui que o sistema precisa ser financiado para ser eficaz, cobrindo os custos elevados dos mesmos. Portanto, foi necessário recorrer a financiamento externo, principalmente através de grandes conglomerados capitalistas, que podem garantir o financiamento necessário para uma maior expansáo para diminuir os elevados custos fixos de microempréstimos. Na consecução deste objectivo, que permite produzir benefícios e capitalizado dependerá em grande parte a sobrevivência deste sistema de financiamento.

Palavras-chave: A pobreza, a necessidade, empoderamento, ação social, o subdesenvolvimento, microempresa

\section{Introducción}

Contra lo que suele creerse, los grupos sociales que se encuentran en los umbrales de la pobreza no solo utilizan mecanismos que pueden considerarse financieros, sino que están, por lo general, acostumbrados a ellos. Es su sistema de financiar inversiones en negocios ocasionales, cubrir las necesidades familiares o los problemas que puede plantear una cosecha escasa.

El problema de los sistemas financieros utilizado por los pobres o los casi pobres no es su inexistencia, sino que son, en la inmensa mayoría de los casos, informales, sin control por un organismo estatal regulador, por lo cual son arriesgados, caros y coyunturales.

A mediados del siglo XX, las entidades de cooperación internacional que solían depender de organismos multinacionales, comenzaron a incluir en sus programas créditos subsidiados dentro de proyectos mucho más amplios.
Lo cierto es que si bien la iniciativa era muy interesante, fracasó debido a una mala planificación económica de la cooperación ${ }^{4}$ y una falta de cultura crediticia por parte de los beneficiarios (Seibel, 2000).

La microfinanciación ha crecido vigorosamente hasta convertirse en una industria sofisticada. En América Latina y el Caribe, unas 600 instituciones de microfinanzas han prestado alrededor de US \$ 12 mil millones a más de diez millones de clientes de bajos ingresos.

En la década de los setenta, grupos solidarios tomaron el relevo y comenzaron a prestar pequeńas cantidades a mujeres para que invirtieran en pequeños negocios. Estas acciones se efectuaron en buena parte como test, especialmente en Bangladesh. Pero no fue hasta finales de la siguiente década -sobre todo a final de siglo-cuando se implementaron programas de microcréditos, lo que mejoró sustancialmente las metodologías para ponerlos en práctica (Soler-Tormo \& Melián-Navarro, 2012).

4. La devolución de los créditos, planificados en función de las cosechas a recoger por los campesinos, no tuvieron en cuenta la posibilidad de un año desastroso para ellas, consecuencia de las abundantes lluvias (Gutiérrez Nieto, 2005). Esta fue una de las causas del fracaso de los créditos rurales, antecedente inmediato de los microcréditos. 
Se establecieron intereses más altos que los ofrecidos por la banca comercial, lo cual se justificaba por la necesidad de rentabilizar las operaciones, debido a que las repercusiones de los costes administrativos en pequeñas cantidades eran porcentualmente más altas. Y, sin embargo, se detectaron desde el principio tasas de devolución mucho más altas que en dicha banca comercial, lo cual condujo a que las microfinancieras, en general, pudieran autofinanciarse y alcanzaran una sostenibilidad que permitió una ampliación de operaciones. El éxito de los microcréditos fue tal que no solo se expandió en países subdesarrollados con grandes bolsas de pobreza o en los umbrales de la pobreza, sino que también se extendió a los países del primer mundo.

Cabe destacar el Banco Palmas, el primer banco comunitario que nació en 1998 en Brasil. La semilla del proceso fue una asociación vecinal que promovió un club de trueque al estilo argentino, el cual evolucionó a un banco comunitario con moneda social y que ahora se plantea dar el salto al dinero electrónico. El principal servicio del Banco Palmas son los microcréditos de dos tipos: al consumo y a la producción. El microcrédito a la producción es concedido en reales -la moneda nacional-y pretende mejorar las redes de relación entre los productores y consumidores, y el destinado al consumo es otorgado en Palmas, la moneda social del banco. El proceso de implantación de la moneda fue lento. La mortalidad de los negocios al inicio era muy alta (la mayoría no superaban los dos años), pero ahora muchas tiendas llevan vendiendo más de diez años. El desarrollo que ha experimentado el comercio local gracias a la moneda ha sido espectacular, al pasar de un $20 \%$ a un $97 \%$.

La primera década del siglo XXI ha visto un desarrollo de este tipo de servicios que han ampliado su oferta de productos financieros. Por otra parte, la sociedad internacional ha comprendido su importancia para sacar de la pobreza a un gran número de personas. Un ejemplo del éxito de los microcréditos y todo el desarrollo a ellos asociado, así como el reconocimiento internacional que ha tenido, es la concesión del Premio Nobel de la Paz de 2006 a Muhammad Yunus, precursor y uno de los mayores impulsores de las microfinanzas. Cierto es que se cometieron errores y a lo largo de su implementación en muchos países han creado problemas, pero la enorme capacidad de las microfinanzas permitió que las capas sociales más desfavorecidas se superaran y alcanzaran la independencia económica (Soler-Tormo \& MeliánNavarro, 2012).

\section{Conceptos y características}

Ragnar Nurske (1953) estableció un paradigma que explica por qué los pobres difícilmente salen de su situación. La práctica totalidad de sus ingresos -dice- los invierten en solucionar sus primeras necesidades, con lo que no pueden ahorrar $y$, consiguientemente, invertir en algún tipo de negocio que les permita mejorar. Por otra parte, su capacidad de garantizar préstamos es nula, razón por la cual no pueden acceder al capital necesario para iniciar una empresa.

Las microfinanzas es el nombre genérico de una serie de herramientas desarrolladas con el objetivo de ayudar a los pobres a salir de su situación. Incluye servicios de préstamo, pero también de ahorro y en la práctica actúa como cualquier banco comercial, solo que sus clientes son otros y sus sistemas de garantías, concesión de créditos y planteamientos de ahorro, diferentes. Las microfinanzas representan una herramienta al servicio de las personas con escasos recursos que les ofrece una variedad de servicios financieros (CGPA5, 2006 y 2007), no solo de crédito, sino también de ahorro y es una de las principales características para la supervivencia del sistema de microfinanzas su sostenibilidad.

Según la pirámide de la pobreza propuesta por Prahalad (2004), será necesario determinar quién es el cliente tipo de las microfinanzas. Por lo general, tanto las organizaciones que actúan sobre el terreno como la doctrina académica, están de acuerdo en dividir a los pobres en cuatro grupos:

\section{Indigentes.}

2. Extremadamente pobres.

3. Moderadamente pobres.

4. Vulnerables, aunque no pobres.

De hecho, las microfinanzas consideran el espectro de la pobreza como un todo que hay que atacar por sectores. Por ello, se han diseñado formas de operar diferentes según las cuatro escalas citadas, de manera que si bien para las categorías tercera y cuarta se exige un tipo de garantías y se

5. El Consultative GrouptoAssistthe Poor (CGAP) o Grupo consultivo de asistencia a la pobreza, es una corporación formada por 33 agencias de desarrollo, tanto públicas como privadas, cuyo objetivo es garantizar, desarrollar y mejorar el acceso de manera permanente a las personas en situación de pobreza a los servicios financieros, especialmente las microfinanzas (www.cgap.org). 
ofrecen cantidades relativamente altas (cuantitativamente) de crédito, para los extremadamente pobres las garantías son más elásticas o se les ofrecen otro tipo de estructuras como las grupales, que veremos más adelante

En los estudios sobre microfinanzas en el caso de los indigentes, siempre se cita un programa dedicado a ellos que implementaron en Bangladesh el Gobierno, el Banco Mundial de Alimentos y el Comité de Fomento Rural, la más importante de las ONG que actúan en el país. Este programa duró diez años y proveyó de ayuda alimentaria a las mujeres en estado de indigencia, al tiempo que les ofrecía servicios de crédito y ahorro. Fue un gran éxito y el $66 \%$ de las mujeres incluidas en dicho programa pasaron al tercer grupo de los moderadamente pobres y entraron en los programas de microfinanzas para crear negocios individuales o familiares que les permitieron salir de la extrema pobreza (Hashemi \& Tudor, 2001).

Una de las características de las microfinanzas estriba en que sus principales clientes son las mujeres, de manera que hay programas solo para ellas (García \& Díaz, 2011). Las causas son varias y dependen mucho de las características sociales de los países, pero uno de los hechos más evidentes es que las mujeres han demostrado un mejor manejo de los créditos, una mayor tasa de devolución y una mayor capacidad para ahorrar (Ledgerwood, 1998). ${ }^{6}$ Hay que tener en cuenta que en la mayoría de estos países, si bien la mujer sufre violencia sexual, también es cierto que es la referencia de la familia.

\section{Microcréditos}

Los microcréditos son pequeños préstamos que se conceden a personas pobres, con tipos de interés por encima de los de la banca comercial y a unos plazos igualmente más cortos que en dicha banca tradicional. Son pequeñas cantidades que se conceden a microempresas formadas en gran número de casos por una sola persona o por un grupo nuclear familiar, en los países en desarrollo (GutiérrezNieto, 2003).

La definición oficial se estableció en la Cumbre del Microcrédito (Washington, 1997):

Se trata de programas de concesión de pequeños créditos a los más necesitados de entre los pobres para que estos puedan poner en marcha pequeños negocios que generen ingresos con los que mejorar su nivel de vida y el de sus familias (Lacalle-Calderón, 2002).
El sistema de microcréditos ha demostrado ser una de las mejores herramientas para erradicar la extrema pobreza en países en desarrollo, dado que si la falta de recursos impide a estos sectores salir de la pobreza, el facilitarles un crédito de acceso fácil, rápido y sostenido les permite salir del círculo vicioso de la pobreza y crear una microempresa que genera riqueza. De manera que el círculo cambie de dirección y la misma dinámica de las microempresas, al generar riqueza, después de cubrir los gastos, posibilita un ahorro remunerado que, a su vez, da lugar a nuevos préstamos y nuevas oportunidades de dejar atrás los niveles de pobreza.

Ahora bien, para que este sistema funcione se dan una serie de condiciones que deben cumplir las partes. En primer lugar, desarrollar una disciplina tanto por parte del cliente como de la institución (Parker \& Pearce, 2001); disciplina del cliente en utilizar el crédito en lo acordado y disciplina de responsabilizarse de que esta inversión implique trabajar y desarrollar el proyecto. También disciplina en el gasto, cumpliendo las cantidades y períodos de devolución acordados. El pago de los intereses y las cuotas convenidas es uno de los puntos neurálgicos de las microfinanzas en general y los microcréditos en particular.

La disciplina por parte de la institución implica el estudio de los intereses que se van a cobrar en cada sector y operación (de ello depende la sostenibilidad del sistema), el seguimiento de dichas operaciones y de las devoluciones. También es su responsabilidad cubrir en lo posible las demandas generadas en el entorno que se mueve y la creación de los productos que puedan necesitar los clientes en el devenir de sus microempresas.

En resumen, los microcréditos son herramientas diseñadas para que los más pobres puedan crear los instrumentos que les permitan salir de la pobreza. Los créditos son pequeños cuantitativamente, pero tampoco se les exigen garantía en la mayor parte de los casos. Frecuentemente se conceden por menos de un año y es este período la duración máxima en la mayoría de las ocasiones. Las tasas de interés son superiores a las de la banca comercial, lo que permite rentabilizar las organizaciones dedicadas a este tipo de finanzas, ya que el hecho de que los clientes sean muchos y de poco volumen encarece el coste de gestión. Consecuentemente, los periodos para el pago de los intereses y la amortización son cortos (Lacalle-Calderón, 2002).

6. El Banco Graneen, fundado por Mohamed Yunus en 1976 y que viene a ser la referencia respecto a instituciones financieras que trabajan en microfinanzas, ha concedido casi el $85 \%$ de sus créditos, los cuales superan en número los dos millones y medio, a mujeres.

$92<$ Universidad de San Buenaventura, Cali - Colombia 


\section{Ahorro}

Queremos incluir un inciso en el análisis de los microcréditos para introducir otro de los servicios que ofrecen las microfinancieras. Cierto que el microcrédito es la estrella del conjunto y que todo parece enfocado hacia el acceso a la financiación de las microempresas que pueden sacar de la pobreza a sus usuarios, pero el ahorro, que parece un logro casi imposible, se ha revelado como uno de los más exitosos productos del sector de las microfinanzas (Mukherjee, 1997).

El problema de los pobres respecto a sus ahorros consiste en dónde depositarlos. Desde luego, los montos son pequeños en función de sus rendimientos, por lo que los bancos comerciales no tienen demasiado interés en crear un producto que pueda satisfacer a los ahorradores pobres. Sí lo han hecho las microfinancieras, que han visto cómo las cuentas de depósito han superado -en algunos casos en mucho- las de préstamos, de modo que se ha revelado que muchos de los moderadamente pobres y los vulnerables pero no pobres, ahorran de manera constante y regular (Mukherjee, 1997).

Podemos observar en la Tabla 1 el número de entidades microfinancieras por categoría y tamaño de las mismas medido por activos totales para el año 2010.

Tabla 1

Activos totales según el tipo de institución microfinanciera (2010)

\begin{tabular}{lcccc}
\hline & $\begin{array}{c}\text { Núme- } \\
\text { ro de } \\
\text { elementos } \\
\text { muestra }\end{array}$ & $\begin{array}{c}\text { Suma activos } \\
\text { totales } \\
\text { (mill \$) }\end{array}$ & $\begin{array}{c}\text { Mediana } \\
\text { activos } \\
\text { totales } \\
\text { (mill \$) }\end{array}$ & $\begin{array}{c}\text { Media } \\
\text { activos } \\
\text { totales } \\
\text { (mil \$) }\end{array}$ \\
\hline Bancos & 100 & 37.525 & 108 & 375 \\
\hline Bancos rurales & 44 & 854 & 11 & 19 \\
\hline Cooperativas & 152 & 4.769 & 5 & 31 \\
\hline IFNB* & 357 & 19.228 & 10 & 53 \\
\hline ONG $^{* *}$ & 412 & 8.374 & 4 & 20 \\
\hline
\end{tabular}

Fuente: Elaboración propia a partir de MIX MARKET (2012b). *IFNB: institución financiera no bancaria.

${ }^{* *} \mathrm{ONG}$ (organización no gubernamental).

La tabla anterior muestra la mediana debido a las grandes variaciones entre los valores máximos y mínimos. De esta manera, la mediana divide a la muestra en dos grupos: la mitad de estas entidades tiene un valor menor de activos totales y el resto un valor mayor.

En cualquier caso, los pobres o moderadamente pobres, para depositar sus ahorros priman una serie de características del producto que se les ofrece: la seguridad y la confianza en la entidad donde depositan sus ahorros.
La tendencia al ahorro de los pobres implica un valor añadido a las microfinanzas. Muchas de las microfinancieras han dejado de apoyarse en las fuentes de financiación estatal o en organizaciones de cooperación y del mercado interbancario para financiarse con el capital acumulado y los ahorros de los pobres.

Sin duda, este tipo de financiación obliga a desarrollar un eficiente sistema de gestión de recursos financieros, de modo que puedan evitarse problemas de liquidez, especialmente en situaciones críticas.

En los niveles de indigentes y de pobreza extrema, el ahorro es muy bajo y se ha orientado más bien a efectos educativos y al ahorro obligatorio (una especie de compromiso mensual de ahorro), aun cuando sean cantidades muy pequeñas. No hay un acuerdo, por cuanto muchos analistas entienden que en primer lugar, es una utopía presuponer que un indigente o un extremadamente pobre puedan drenar una parte, aunque sea ínfima, de sus recursos para ahorrar; y en segundo lugar, que si cualquiera dentro de los dos niveles solicita un crédito este será pequeño y necesitará todos los recursos no solo para vivir él y su familia, sino también para reinvertir en el micronegocio o la microindustria que haya creado. Por lo que, por el momento, la obligación de ahorrar no se ha generalizado (Westley, 2003)

\section{Características y funciones de los microcréditos}

Como ya hemos dicho, la principal función de los microcréditos es la lucha contra la pobreza a través de la concesión de pequeños créditos a personas que, por sí mismas, no tienen posibilidades de acceder a cualquier financiación comercial, y que piensan utilizarlos para crear mecanismos económicos, microempresas o micronegocios, que les permitan mejorar su nivel de vida y acceder -ellos o sus hijos- a la educación o a la sanidad, entre otros avances sociales (Lacalle-Calderón, 2002).

Se plantea una primera disyuntiva y es la siguiente: si el microcrédito debe entenderse como un fomento a la producción o una lucha por la supresión de la pobreza. La respuesta al parecer depende de la situación del entorno en que se implementa; por otra parte, las dos posturas pueden llegar a ser complementarias. A veces el problema planteado es social, el desempleo es un ejemplo. Se trata, entonces, de fomentar el empleo sobre la base de crear microempresas o micronegocios que permitan unos ingresos para mantener la familia. En otro orden de ideas, la falta de capacitación implica la necesidad de un microcrédito que 
permita capacitarse o capacitar un grupo para competir en el mercado produciendo un producto competitivo.

Los estudios confirman que el microcrédito se ha convertido en una herramienta fundamental para el desarrollo de las personas y las pequeñas unidades productivas, permitiendo así que muchas de ellas tengan acceso por primera vez al sistema financiero. El Fondo Multilateral de Inversiones (Fomin), miembro del Banco Interamericano de Finanzas (BID), publicó recientemente el informe Microfinanzas en América Latina y el Caribe: el sector en cifras, en el cual presenta referencias cuantitativas sobre la evolución de dicho sector desde el año 2001 al 2012.

Según el peso de la cartera de microcréditos en países de América Latina y el Caribe, se aprecia que si bien Brasil cuenta con el portafolio más grande, su participación dentro de su sistema financiero es ínfimo (1,3\% de la cartera total de créditos). En Bolivia o Ecuador el peso es mucho más significativo (35,2\% y 20,7\%, respectivamente). En Bolivia el microcrédito desempeńa un papel fundamental como motor de la economía local. Esta afirmación se desprende luego de observar el ratio microcréditos/PBI para el 2012, el cual llegó al 13,7 \%. En Ecuador, la participación de los microcréditos dentro de su sistema financiero se situó en $20,7 \%$, aunque el tamaño de dicho portafolio con respecto al PBI es de solo 3,3\%. Para el caso peruano, el peso del microcrédito, tanto del sector regulado como no regulado dentro del sistema financiero, llegó al $12,4 \%$, mientras que con respecto al PBI se ubicó en $4,2 \%$.

Indudablemente, el microcrédito necesita que allí donde va a ser aplicado se dé previamente un cierto nivel de actividad económica y capacidad de innovación; o sea, capacidad para crear empresas y promocionarlas. Es necesario también un cierto mercado. En cualquier caso, dado el tipo de industrias o negocios que crean y desarrollan los pobres y los mercados potenciales a los que pueden dirigirse -que en general son locales- no es frecuente que se encuentren con problemas más allá de las de acceso al microcrédito.

Este acceso puede hacerse de muy diversas formas, aunque generalmente son cinco las vías más comunes: los grupos solidarios, las uniones de crédito, los bancos comunales, los fondos rotatorios y los microcréditos individuales.

Estas vías operan, por lo general, solapándose en el mismo entorno y dependen más de la naturaleza del cliente que del tipo de microempresa que se quiera desarrollar. Aunque cada una de estas vías tiene características propias, diferentes unas de las otras, cumple también con objetivos específicos y diferentes de los demás.

\section{Grupos solidarios o colectivos}

Los microcréditos son concedidos a grupos de personas que responden de manera solidaria del total entregado o son créditos individuales, pero avalados por el resto de los miembros del grupo que responden solidariamente por ellos.

La principal ventaja de este sistema estriba en que llega a personas en estado de extrema pobreza que difícilmente por sí mismos, sin la solidaridad de los otros miembros del grupo, podría acceder a los microcréditos o con dificultades para cubrir los repagos sin ayuda. Aun cuando también puede ser por razones exógenas, como que viven en zonas rurales a las que no llegan los servicios de las microfinancieras.

El monto de las operaciones no suele superar, en la mayor parte de los casos, los 500 dólares y suele estar entre 100 y 250 , aun cuando las cantidades son bastante variables y dependen del estudio que hace la microfinanciera del caso. Asimismo es frecuente que se obligue a una tasa de ahorro determinada, generalmente una parte del crédito que actúa a la vez como garantía y crédito, puesto que si se han cumplido las condiciones de devolución, suele renovarse y ampliarse (Karlan \& Golberg, 2011).

\section{Uniones de crédito}

Según el instituto Zabala (2014), las uniones de crédito, antecedentes de las actuales cajas de ahorro europeas, aparecieron en la década de los cincuenta en los países en desarrollo y derivan de la asociación voluntaria de personas que comparten determinados lazos geográficos o actividades afines (mismo sector, misma fábrica) que se agrupan para poner sus ahorros en común y proporcionar créditos a unas tasas de interés razonables.

Como cooperativas de ahorro y crédito (Lacalle, 2010), no tienen ánimo de lucro y están gestionadas democráticamente por sus miembros. Para recibir un crédito es necesario mantener una cuenta de ahorro en la cooperativa, por lo que es una metodología incluida dentro de las de de ahorro forzoso y por lo tanto autosuficiente. Son, además, instituciones locales formadas por agentes del entorno que utilizan sus recursos, característica que comparten con los bancos comunales.

Las garantías que exigen son mínimas y están basadas fundamentalmente en la cuenta de ahorro que obligatoriamente se tiene en la propia cooperativa, así como en las relaciones entre los miembros de la cooperativa. 
Tabla 2

Metodologías del microcrédito

\begin{tabular}{|c|c|c|c|c|}
\hline Grupos solidarios & Préstamos individuales & Uniones de crédito & Bancos comunales & Fondos rotatorios \\
\hline $\begin{array}{l}\text { Grupo de entre } 5 \text { y } 8 \\
\text { individuos. }\end{array}$ & Individual. & Grupo & $\begin{array}{l}\text { Grupo entre } 30 \text { y } 50 \\
\text { personas. }\end{array}$ & $\begin{array}{l}\text { Tienen su origen en las } \\
\text { ROSCAS1 }\end{array}$ \\
\hline Garantía mutua. & Garantía individual. & Garantías muy reducidas. & Garantía mutua & \\
\hline $\begin{array}{l}\text { Autosuficiencia finan- } \\
\text { ciera. }\end{array}$ & & Autosuficiencia financiera. & $\begin{array}{l}\text { Autosuficiencia finan- } \\
\text { ciera. }\end{array}$ & Autosuficiencia financiera. \\
\hline Proceso sencillo y rápido. & Proceso sencillo y rápido. & Proceso sencillo y rápido. & Proceso sencillo y rápido. & Proceso sencillo y rápido. \\
\hline $\begin{array}{l}\text { Requisito: microem- } \\
\text { presa o capacidad para } \\
\text { emprender una actividad } \\
\text { económica. }\end{array}$ & $\begin{array}{l}\text { Requisito: microempresa o } \\
\text { capacidad para emprender } \\
\text { una actividad económica. }\end{array}$ & $\begin{array}{l}\text { No requisito; cubren } \\
\text { cualquier necesidad de los } \\
\text { miembros. }\end{array}$ & $\begin{array}{l}\text { Requisito: microem- } \\
\text { presa o capacidad para } \\
\text { emprender una actividad } \\
\text { económica. }\end{array}$ & \\
\hline $\begin{array}{l}\text { Reduce los costes } \\
\text { medios. }\end{array}$ & $\begin{array}{l}\text { El monto de estos créditos } \\
\text { es mayor que el del resto. }\end{array}$ & & $\begin{array}{l}\text { Proporcionar servicios de } \\
\text { ahorro y crédito indivi- } \\
\text { duales a los miembros. }\end{array}$ & \\
\hline \multicolumn{5}{|l|}{$\begin{array}{l}\text { Opera de forma descen- } \\
\text { tralizada. }\end{array}$} \\
\hline $\begin{array}{l}\text { Se ajusta a las necesida- } \\
\text { des de sus clientes y tiene } \\
\text { en cuenta sus preferen- } \\
\text { cias. }\end{array}$ & $\begin{array}{l}\text { Se adapta a la capacidad de } \\
\text { pago y a las características } \\
\text { del cliente. }\end{array}$ & & $\begin{array}{l}\text { Se ajusta a las necesidades } \\
\text { de sus clientes y tiene en } \\
\text { cuenta sus preferencias. }\end{array}$ & $\begin{array}{l}\text { Los fondos no siempre se } \\
\text { adaptan a las necesidades } \\
\text { del cliente, dado que cada } \\
\text { miembro debe esperar su } \\
\text { turno. }\end{array}$ \\
\hline
\end{tabular}

Formado principalmente

por mujeres.
La mayoría de los miem-

bros son mujeres.

Los miembros reciben Los miembros reciben capa- No se promociona capacita- Los miembros reciben capacitación y asistencia. citación y asistencia. ción ni asistencia. capacitación y asistencia.

Fuente: Torre-Olmo, Sainz, San Filippo y López (2012).

Su principal limitación es la falta de creación de nuevo capital para ampliar su capacidad crediticia. Ello es debido a su reducido tamaño, cobro de intereses por debajo del de mercado y alta retribución a los ahorros de sus cooperativistas (Lacalle-Calderón \& Rico-Garrido, 2008).

\section{Bancos comunales}

Se trata de una asociación de personas de una misma comunidad o comunidades muy próximas que se agrupan para autogestionar, desarrollar y promover el ahorro y el crédito. ${ }^{7}$ Suelen ser grupos de veinte a cincuenta personas que pertenecen a comunidades rurales más o menos aisladas, aun cuando pueden darse también en núcleos urbanos.

Los miembros del grupo eligen un comité que se encarga de la gestión. La financiación del banco se inicia con una aportación de los miembros que pasa a consolidarse como un ahorro, cuyo monto estará relacionado con el montante global de crédito que puede obtenerse. Por otra parte, los miembros del grupo son garantes de los créditos de forma mancomunada, con lo que se logra que sean los propios miembros los que presionan a sus compañeros para que cubran su parte alícuota del crédito y de los intereses. Las tasas de interés varían según el tipo de crédito y la garantía -que en ocasiones puede ser la propia cosecha- presentada.

La retribución de este ahorro se reparte proporcionalmente al ahorro efectuado por los cuentaparticipes. Por otra parte, los créditos suelen ser de menor cuantía, generalmente entre 100 y 150 dólares, y los plazos de seis a doce meses (Westley, 2003). ${ }^{8}$

Una de las justificaciones que se dan para la adopción de este modelo de organización del microcrédito es el

7. La propuesta fue de John y Margarita Hatch en los años ochenta y derivó en la creación de la Fundación para la Asistencia Comunitaria Internacional (Finca)

8. Hay que tener en cuenta que estas cifran han de considerarse en el país y zona específica del país del que se trata. Cien dólares en Bangladesh o incluso en zona de países iberoamericanos es una cantidad alta y suficiente para invertirlos en una explotación agraria o de otro tipo y sacar rendimientos que justifiquen la inversión. 
ahorro que supone para las microfinancieras hacer una sola operación de crédito en vez de cincuenta. Sin embargo, los estudios sobre la fórmula no dicen lo mismo. Según Westley (2003), que trabaja con datos de Finca y del Microbanking Bulletin, si se analiza la cuestión desde la perspectiva de todas las entidades microfinacieras, es cierto que los bancos comunales ahorran en la gestión por cuanto sus empleados de cuentas controlan 350 clientes de promedio por 275 de las entidades microfinancieras. Sin embargo, si se comparan solo las entidades microfinancieras sostenibles y los bancos comunales sostenibles financieramente, los empleados de cuentas de las primeras controlan 425 clientes por 290 de los segundos. El tipo de entidades que se dedican a este sector de las microfinanzas va desde entidades microfinanceras típicas hasta ONG, pasando por entidades estatales o paraestatales y hasta bancos comerciales.

El éxito de los bancos comunales no se centra, entonces, en el crédito, sino en otros servicios, especialmente el ahorro, los cursos de formación, los beneficios que comporta el empoderamiento y la asistencia técnica que proporciona.

\section{Fondos rotatorios}

No son tanto un tipo de microfinanzas como un sistema de cooperación mutua. Son grupos con intereses comunes que se forman a nivel local y acuerdan aportar una cantidad mensual o semanal que, tras estructurar un sistema de turnos, se entrega a uno de los miembros. Naturalmente, este miembro se compromete a la devolución en el plazo establecido y con los intereses indicados. El sistema de devolución es diverso y va desde el mencionado, -devolución del principal más intereses- hasta el caso en el que el beneficiario se queda con la cantidad y no está obligado más que a continuar pagando la cuota semanal o mensual (CGAO, 2006). ${ }^{?}$

Este sistema puede implementarse en una zona rural o urbana, entre grupos de mujeres, hombres o mixtos, con similares o diferentes rentas y con iguales, aunque deben tener intereses comunes y una idea clara sobre sus obligaciones contractuales. Otro de los problemas radica en que cualquier miembro debe esperar a que le llegue su turno, aun cuando necesite el crédito antes (Villarraga, 2008).

\section{Microcréditos individuales}

Es el microcrédito tipo y, consecuentemente, el más usado y conocido. Una parte de la doctrina lo define como un híbrido entre el crédito tradicional y el que siguen los prestamistas. Es decir, por una parte la entidad microfinanciera analiza los flujos de caja, los avales y los estados financieros del solicitante; pero además, se estudia también la unidad familiar, el entorno del cliente, la microempresa que quiere fundar o desarrollar y evalúan al cliente por el conocimiento directo que tienen de él y ha sido adquirido por las relaciones directas.

Esto implica que el empleado encargado de esta cuenta tenga un trato personal con sus clientes, los conozca y conozca a su familia. Naturalmente esto limita el número de clientes que puede atender, que por lo general van de sesenta a ciento cuarenta, aunque depende mucho de los entornos. Este sistema se ha demostrado especialmente eficiente respecto a la devolución de principal e intereses (Gutiérrez-Nieto, 2003).

En este tipo, el monto de los créditos es muy variable. Puede ir de los cien a los tres mil dólares y está en función del cliente y sus posibilidades de devolución. También los plazos de retorno pueden ser cortos o largos: desde seis meses o menos a cinco ańos. La capacidad del cliente de invertirlo y su historial respecto a préstamos anteriores, así como sus garantías, condicionan mucho que el crédito sea alto y a un plazo largo.

Las microfinancieras tienden a préstamos a largo plazo y a cantidades grandes. Los costes de gestión son menores. Sin embargo, la mayoría de las peticiones son de créditos de muy poca cantidad y a plazos cortos, con pagos semanales (Karlan \& Golberg, 2011).

\section{Impacto de los microcréditos}

Como hemos dicho, los objetivos de las microfinanzas en general son erradicar la pobreza a través de la recuperación económica de los más necesitados y lograr que tengan acceso a los servicios de salud, educación y promoción básicos. Ello implica una mejora de la renta familiar, la seguridad de una atención sanitaria suficiente y la posibilidad de que los hijos tengan, a través de la educación, un mejor futuro.

9. Este tipo de agrupaciones se han utilizado en momentos de crisis económica y financiera para comprar bienes de consumo como, por ejemplo, coches, televisiones o incluso ropa o comida, o incluso puede funcionar como un sistema de ahorro. En Argentina fueron muy populares durante las crisis que se sucedieron a finales del siglo pasado. 
Aunque los movimientos de cooperación en general y las microfinancieras en particular aún no han logrado estos objetivos en toda su plenitud, están en el camino correcto para lograrlo. ${ }^{10} \mathrm{Y}$ cuando citamos a las microfinancieras nos referimos a todas las entidades que de una manera u otra están implicadas en la promoción a través de los microcréditos a los más pobres.

Un ejemplo del impacto de los microcréditos en una zona donde están muy implantados todos los tipos antes enumerados (América Latina), son las estadísticas relativas a 2011. El total de instituciones que se dedican a los microcréditos es de 605 (169 reguladas y 436 no reguladas), con una cartera de créditos de 18.917 millones de dólares (15.492 millones de dólares para las no reguladas y 3.425 para las reguladas) con un total de 14.942.357 (10.498.505 para las reguladas y 4.443 .772 para las no reguladas) y con un crédito promedio de 1.476 dólares para las institución reguladas y 771 para las no reguladas.

Las cifras que explican la evolución de los microcréditos en América Latina en la última década son las comparativas entre el 2001 y el 2011. En el 2001, el total de las instituciones microcrediticias era de 184 con 1.189 millones de dólares de cartera, 1.806 .445 clientes y un crédito promedio de 659 dólares. En 2011, había casi cuatro veces más de entidades microfinancieras que diez años antes; el número de clientes se había multiplicado por diez y la cartera de clientes por casi dieciséis. Pero también es importante destacar que el crédito promedio fue casi el doble que en 2001. El aumento del crédito promedio se debe, en su mayor parte, a los clientes que devolvieron según las condiciones el primer crédito y han solicitados otros superiores para ampliaciones del negocio, compra de materia prima en condiciones ventajosas o la adquisición de maquinaria (Pedroza, 2012).

Si bien es cierto que es muy difícil evaluar estas cifras en el largo plazo, también lo es que indican cuanto menos una tendencia. Así, Khandker-economista del Banco Mundial que elaboró un conocido informe sobre el impacto de programas de ayuda en Bangladesh y la importancia del factor sexo en ellos- evaluó el impacto de tres de los más importantes programas de microfinanzas implementados en Bangladesh en un $40 \%$ de la reducción de la pobreza moderada en dicho país (Goldberg, 2005). ${ }^{11}$

Son ejemplos de cómo los microcréditos impactan sobre las colectividades en las que se asientan. Por un lado, aumentan la renta disponible en la familia y permiten acceder a beneficios, que van desde nuevos consumos hasta la compra de tierras o ganado que aumenten la riqueza familiar; además de hacer frente a situaciones de crisis que puedan aparecer. Por otra parte y respecto al entorno más próximo, el barrio, el pueblo o la comarca, aumentan las posibilidades de desarrollo industrial y empresarial, lo que contribuye a un aumento de los puestos de trabajo y el circulante, creando así un efecto positivo en la economía de dichos colectivos.

No puede negarse que han continuado presentándose problemas durante la primera década del siglo XXI: aumentos de costes paralelos a los aumentos de peticiones de créditos, y los Estados continúan con su política de subsidios para solucionar crisis coyunturales y problemas de buen gobierno de las propias entidades microfinancieras, entre otros (Demirgüç-Kunt, Beck \& Honohan, 2007; Ditcher \& Harper, 2007; Lescelles, 2008). Sin embargo, en conjunto las microfinanzas en general y su producto más representativo, a saber, los microcréditos, se han revelado como el mejor y más eficiente sistema para reducir las bolsas de pobreza no solo en los países subdesarrollados, sino también en los entornos más desfavorecidos del primer mundo en los que el microcrédito se ha introducido en los últimos diez años con notable éxito.

Si el pasado de las microfinanzas estuvo plagado de errores en función de la intervención de los Estados y el presente está cumpliendo los objetivos que se planteó en buena parte, el futuro se presenta lo suficientemente positivo como para tener fundadas esperanzas en el sistema.

\section{Conclusiones}

Las microfinanzas a finales del siglo XX fueron la consecuencia evolutiva de las agencias de cooperación que instituyeron mayoritariamente los Estados, los organismos internacionales y las organizaciones particulares para ayudar a los más pobres en los países más pobres.

La eclosión de las microfinanzas y de sus diversos productos se produce en la última década del siglo XX y primera del XXI, especialmente en el sudeste asiático, países de Oriente Medio y América Latina. Propuestas como el Banco Grameen del Premio Nobel Mohamend Yunus, en Bangladesh, y la difusión de las microfinanzas en países como Egipto y Marruecos, así como en América

10. No obstante también hay voces críticas con respecto al papel de las microfinancieras y los microcréditos como panacea para erradicar la pobreza. Véase por ejemplo el trabajo de Martínez (2008) en este sentido.

11. Los programas fueron el BRAC, Banco Grameen y RD-12. 
Latina y el Caribe donde se mantiene pujante y continúa su expansión en la última década al experimentar un aumento en su número de clientes, una gran variedad de instituciones, y una tendencia a la baja en las tasas de interés, han marcado las nuevas tendencias del sistema.

Es un sistema que se fundamenta en pequeños créditos -en muchos casos de no más de 150 dólares-que se otorgan a pobres para iniciar un negocio que, por lo general, se trata de una industria individual como, por ejemplo, la compra de una máquina de coser para elaborar trajes o piezas de ropa, un carro para repartir o una bicicleta para convertirse en mensajero. Se trata de salirse, en una primera fase, del paro y encontrar una fuente de ingresos.

Se trata de crear las bases de una economía social y financiarla. Esta financiación puede hacerse a través de ONG, cooperativas, microfinancieras independientes, bancos especializados $\mathrm{u}$ otro tipo de organizaciones $\mathrm{y}$ entidades, cada una con sus peculiaridades estructurales e incluso el mercado al que se dirigen, pero los objetivos sociales son los mismos o muy parecidos.

El sistema necesita autofinanciarse para ser efectivo. Las tasas de interés son altas y permiten esta autofinanciación, pero solo cuando se ha logrado una fluidez en los retornos y pago de intereses. Las tasas de retorno son muy altas y el sistema comercial es sólido, pero los costos también lo son. Adecuar los costos a los rendimientos para cubrir la gestión, producir beneficios y capitalizarse ha sido uno de los más importantes objetivos de esta última década. Una solución ha sido la financiación externa: estados y agencias de cooperación, pero por lo general a cambio de subsidiar los créditos, lo cual ha dado casi siempre resultados negativos.

La entrada en el sector de los bancos comerciales y la expansión de las microfinanzas a los países del primer mundo, han cambiado las expectativas del futuro tanto respecto a los mercados como a los agentes que van a intervenir con más fuerza en la próxima década. Pero al tiempo que esto acerca las microfinanzas a los grandes conglomerados capitalistas, garantiza que recibirán la financiación necesaria para su mayor expansión. Este es el futuro que prevemos para las microfinanzas.

\section{Referencias}

Consultative Group to Assist the Poor. (2006). Guidelines for Funders of Microfinance. WA. Recuperado de https:/goo. $\mathrm{gl} / \mathrm{LfuDux}$
Consultative Group to Assist the Poor. (2007). Annual report. WA. Recuperado de http://goo.gl/eNq0La

Demirgüç-Kunt, A., Beck, T., \& Honohan, P. (2007). Finance for all? policies and pitfalls in expanding access. WA: World Bank Publications.

Ditcher, T., \& Harper, M. (2007). What's wrong with microfinance. Rugby: Practical Action Publishing.

García, F., \& Díaz, Y. (2011). Los microcréditos como herramienta de desarrollo: revisión teórica y propuesta piloto para el África subsaharian. Revista de Economía Pública, Social y Cooperativa, 70, 101-126.

Goldberg, N. (2005). Measuring the impact of microfinance: Taking stock of what we know. WA: Grameen Foundation.

Gutiérrez-Nieto, B. (2005). Antecedentes del microcrédito. Lecciones Del pasado para las experiencias actuales. Revista de Economía Pública, Social y Cooperativa, 51, 25-50.

Hashemi, S., \& Tudor, M. (2001). Linking microfinance and safety net programs to include the poorest: the case of IGVGD in Bangladesh. CGAP Focus Note, 21, 1-12.

Zabala, N. (2014). Microcréditos. Recuperado de http://goo. $\mathrm{gl} / 9 \mathrm{TY} 5 \mathrm{a}$

Karlan, D., \& Goldberg, N. (2011). Microfinance Evaluation Strategies: Notes in Methodology and Findings. In B, Armendáriz \& M. Labie (Eds.), The Handbook of Microfinance. London: World Scientific Publishing.

Lacalle-Calderon, M. (2002). Microcréditos. De pobres a microempresarios. Barcelona: Ariel.

Lacalle-Calderon, M., \& Rico-Garrido, S. (2008). Microcréditos y pobreza: de un sueño al Nobel de la Paz. Madrid: Ediciones Turpial.

Lacalle-Calderon, M. (2010). Glosario Básico sobre Microfinanzas. Madrid: Foro Nantik Lum de Microfinanzas.

Ledgerwood, J. (1998). Microfinance Handbook. WA: The World Bank.

Lascelles, D. (2008). Microfinance Banana skins. NY: CSFI.

Nurske, R. (1953). Problemas de formación de capital en los paises insuficientemente desarrollados. México: Fondo de cultura económica.

Martínez, A. D. (2008). El microcrédito como instrumento para el alivio de la pobreza: Ventajas y limitaciones. Cuadernos de Desarrollo Rural, 5(61), 93-110.

Parker, J., \& Pearce, D. (2001). Microfinanzas, donaciones y respuestas no financieras para la reducción de la pobreza: ¿Dónde encaja el microcrédito? CGAP, Enfoques, 20, 1-11.

Pedroza, P. A. (2012). Microfinanzas en América Latina y el Caribe: El sector en Cifras. Madrid: Fondo Multilateral de Inversiones. 
Prahalad, C. K. (2004). The Fortune at the Bottom of the Pyramid: Eradicating Poverty through Profits. NY: Wharton School Publishing.

Mukherjee, J. (1997). Introducing Savings in Microcredit Institutions: When and How? CGAP Focus Note, 8, 1-8.

Seibel, H. D. (2000). From cheap credit to easy money: How to undermine rural finance and development. In F.J.A. Bouman $\&$ O. Hospes (Eds.), Financial Landscapes Reconstructed (pp. 19-32). CO: Westview Press.

Soler-Tormo, F., \& Melián-Navarro, A. (2012). Cooperativas de crédito y banca social: viejas y nuevas respuestas éticas y solidarias a problemas de siempre. Revesco, 109, 45-80.
Torre-Olmo, B., Sainz, I., San Filippo, S., \& López, C. (2012). Guía sobre Microcréditos. Santander: Universidad Cántabra.

Villarraga, J. (2008) Fondos autogestionados rurales de ahorro y crédito. Experiencia y lecciones para el fortalecimiento de las microfinanzas rurales de Colombia. Bogotá: Corporación para el Desarrollo Comunitario.

Westley, D. (2003). Relato de cuatro programas de banca comunal. WA: Banco Interamericano de Desarrollo. 
